Mathematical Modelling and AnAlysis

Volume 17 Number 1, February 2012, 31-46

http://dx.doi.org/10.3846/13926292.2012.644406

(c) Vilnius Gediminas Technical University, 2012
Publisher: Taylor\&Francis and VGTU

http://www.tandfonline.com/TMMA

Print ISSN: 1392-6292

Online ISSN: 1648-3510

\title{
The Mathematical Modelling of Liner Movement in a Magnetic Compressor: Elastic, Liquid and Plastic Liner Models Comparison
}

\author{
Michail Galanin ${ }^{a}$, Aleksey Lototsky ${ }^{b}$ and \\ Alexander Rodin ${ }^{a}$ \\ ${ }^{a}$ Keldysh Institute of Applied Mathematics of RAS \\ Miusskaya sq. 4, 125047 Moscow, Russia \\ ${ }^{b}$ SRC RF TRINITI \\ 142190 Troitck, Moscow region, Russia \\ E-mail: galan@keldysh.ru \\ E-mail(corresp.): rals@bk.ru
}

Received September 1, 2010; revised October 14, 2011; published online February 1, 2012

\begin{abstract}
The paper is aimed to model the electromagnetic acceleration and braking of the liner in magnetic compressor. The $2 \mathrm{D}$ approach corresponding to the longitudinal section of spatial region is considered. Liquid, elastic, and plastic models of the liner are presented. The comparative analysis of calculation results for different models and their correlation with experimental data are carried out. The research of the influence of circuit parameters on liner braking is done.
\end{abstract}

Keywords: modelling, electrodynamics, large deformations.

AMS Subject Classification: $81 \mathrm{~T} 80$.

\section{Introduction and Statement of the Problem}

The unit "MOL" ("Magnetic Oblateness of Liners") is aimed to study the operation of all stages of the "Baikal" unit module and the generation of electrical pulses of megajoule magnitude. The model of a power sharpening cascade, i.e. the magnetic compressor (MC), have been developed for the "MOL" unit [1]. The operation of the cascade is based on the compression of a magnetic flow by a liner accelerated by electromagnetic forces till the velocity $1 \mathrm{~km} / \mathrm{sec}$. The sketch of compressor's basic details (the copper inductor and the liner) is presented in Fig. 1a. The liner is the aluminum belt which is strained by the tensioning device into a couple of parallel bands. The liner has the following typical sizes: the belt thickness (axial $x$ length) is $2 \mathrm{~mm}$, the belt width (axial $y$ length) is about $21 \mathrm{~cm}$, axial $z$ belt length is about $80 \mathrm{~cm}$, the distance between the parallel belts at the start time is about $20 \mathrm{~cm}$. The MC is presented in Fig. $1 b$. 

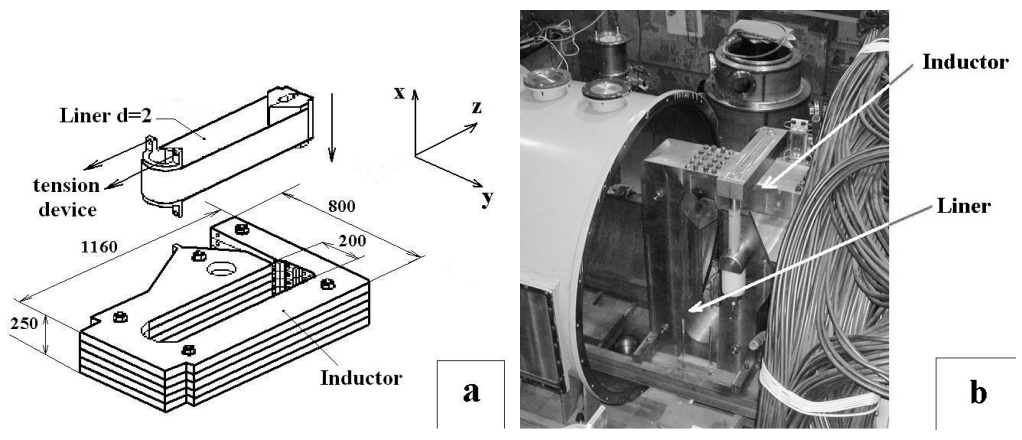

Figure 1. (a) the sketch of the MC; (b) the MC photo.

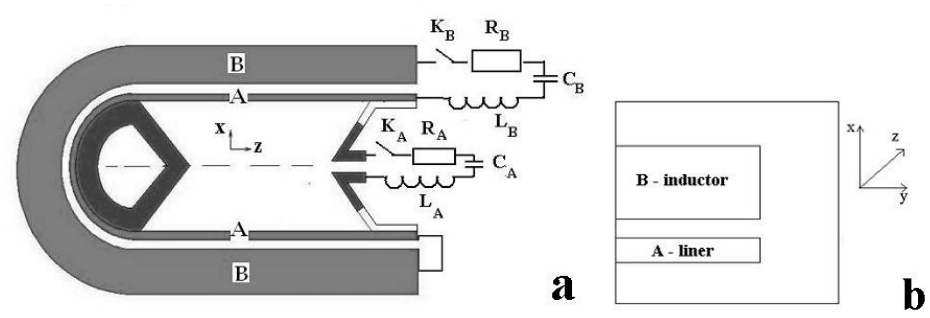

Figure 2. (a) the scheme of the longitudinal section (at the plane $y=$ const); (b) the scheme of a quarter of the transverse section (at the plane $z=$ const).

In the implosive-magnetic generator the work zone is placed within accelerated shell. In the MC it is necessary to connect the liner and the fixed conductor lines to output the current to the external load. It stipulates the choice of the MC sample with the double strip conductor. The production of the liner belt is quite simple process. The design of prismatic supports situated at both compressor ends (Fig. 2a) provides the contact between the liner and the fixed conductor lines. These supports also prevent the belt and current circuit of the liner from breaking.

The sections of the MC at the planes $y=$ const (longitudinal section) and $z=$ const (transverse section, one fourth of the whole section) are shown in Fig. 2. The electrical inductor and liner circuits which can be interconnected are presented. in these figures. Here and hereinafter, liner, inductor and values referred to them are indicated with the indices $A$ and $B$.

At the initial time moment the capacitor $C_{B}$ (in the inductor circuit) is charged to a certain initial voltage. After the closing of the circuit by the switch $K_{B}$, the discharge current begins to flow via inductor and liner. The produced magnetic field interacts with the current running via the liner, thus accelerating the liner along the $x$-axis. The left prismatic support (Fig. 2a) is a conductor, the right support is a non-conductor, but it has the conductive plate situated in the center part of the support. The liner in motion is pressed against the 
supports and when the liner belt comes into contact with this conductive plate the liner circuit is closed. The liner current flows via the belt and produces an additional magnetic field inside the liner cavity. Having been accelerated the liner compresses the magnetic field, which is taken from the system in the form of the current pulse in the external liner circuit. The specific parameters for $\mathrm{MC}$ are the following: the liner velocity is about $1 \mathrm{~km}$ per second, the acceleration time is about 100-150 microsecond, the deceleration time is about 1 microsecond, the circuit current is equal to several mega-ampere.

The work has been done under partial financial support of the Russian Fund for Basic Researches (projects N 09-01-00151 and N 10-08-01308).

\section{The Experimental Data. The Actuality of Mathemati- cal Modelling Application}

Prior to designing and creating a full-scale power it is necessary to know the scale of probable heterogeneities that occur during the acceleration and braking of a flat liner with limited dimensions in a magnetic field. This was the reason for creating the MC prototype in SRC RF TRINITI, Troitsk, which is shown in Fig. 1b.
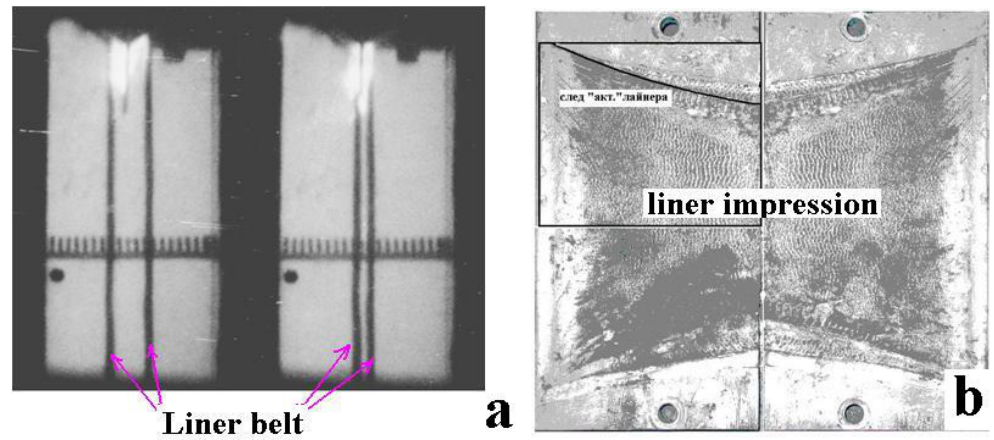

Figure 3. (a) the shots of liner movement (119, 134 microsecond); (b) the liner impression situated on the surface of prismatic support.

A number of experimental launchings was carried out in the MC prototype, but the acquired information is insufficient because of the experiment fleetness and experimental conditions. The frames of shaded camerawork are presented in Fig. 3a (see [5] for more information). These frames demonstrate that the liner belts are moving in a plane-parallel motion and their central parts do not bend in the longitudinal section of MC.

The experimental data of the effective liner width are also presented in the paper [5]. These data are obtained from the measurement of the liner impression situated on the surfaces of prismatic supports. This impression characterizes the liner movement in the transverse section of the MC. The boundaries of the deformed liner belt are seen well in Fig. 3b. The belt width decreases while liner is moving. The belt narrowing is probably caused by the 
liner edge twisting provoked by the pinch effect. But it appears that the liner acceleration heterogeneity starts the process.

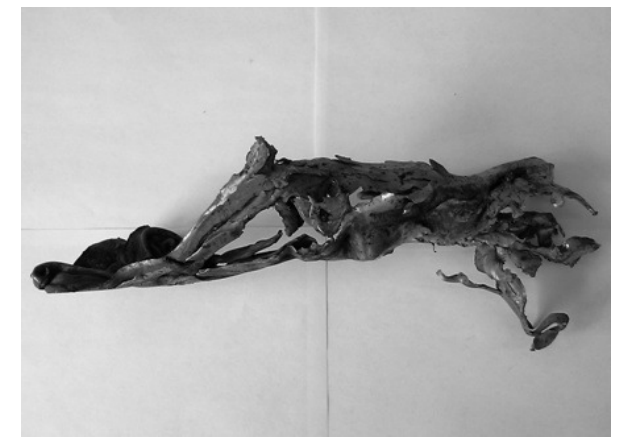

Figure 4. A liner fragment after experimental launching.

The analysis of deformed liner belt after MC experimental launching can make clear the deformation process features but the most part of the belt is destroyed at the launching end. The photo of one liner fragment is presented in Fig. 4. The regime of the magnetic flow compression requires the strict geometry form of the gap between the belts at the moment of liner braking. The generated short pulse needs the simultaneous transformation of the kinematic energy of the whole liner into the output pulse energy. The deformation of the flat form leads to the in-coordinate braking of different liner parts and to the output pulse stretching.

The experimental data confirm the existence of nonhomogeneities during the liner movement but the experiment is not able to determinate the cause and to make the exact quantitative analysis of the liner deformation process. For this reason mathematical modelling and computational experiment are the exclusive methods to obtain more detailed information about liner movement in MC. Furthermore computational experiment is a flexible and convenient tool for executing a number of optimization calculations with the aim to select optimal option values of MC.

The objective of this work is the development of different mathematical and numerical models describing liner movement in the $\mathrm{MC}$, comparative analysis of the calculation results and their correlation with the experimental data.

\section{The Mathematical Models}

The described process is a complicated one therefore different spatial twodimensional approaches are used. The longitudinal model corresponds to the longitudinal section (Fig. 2a), all variables for this model do not depend on $y$. The transverse model corresponds to the transverse section (Fig. 2b), all variables for this model do not depend on $z$. The system effective length along the third axis (accordingly $l_{y}$ and $l_{z}$ ) is taken into account. This parameter is 
determined by the transformation of energy from the electric circuit energy to the liner kinetic energy.

Each model has its own advantages and disadvantages. The longitudinal model does not consider transverse deformations but it permits us to describe the liner movement near the prismatic supports. This region is a source of possible nonhomogeneities. The transverse section determines the basic configuration of compressor magnetic field, but the corresponding model does not consider the contact between the liner belts and the prismatic supports. While correlating and comparing these models it is possible to make conclusions about real processes that take place in the three dimensional system. In this work the longitudinal model is considered unless otherwise stated. The transverse model is presented in detail in papers $[5,6,9]$.

The inductor and the prismatic supports are assumed to be fixed, their shapes are constant. The inductor is a conductor, the rarefied air in the MC box is assumed to be the vacuum.

\subsection{The electrodynamic problem}

The electrodynamic model is based on a system of the Maxwell equations in magneto-hydrodynamic approximation (demonstrated in dimensionless form, $[10,14])$ :

$$
\begin{gathered}
\frac{\partial \mathbf{H}}{\partial t}=\nabla \times([\mathbf{v} \times \mathbf{H}]-\mathbf{E}), \\
\nabla \times \mathbf{H}=4 \pi \sigma \mathbf{E}=4 \pi \mathbf{j}, \\
\nabla \cdot \mathbf{H}=0 .
\end{gathered}
$$

Here $\mathbf{E}$ is an electric field intensity (in the system of axes where material does not move), $\mathbf{H}$ is magnetic field intensity, $\mathbf{v}$ is a velocity vector of medium points movement (liner points in this particular case), $\sigma$ is a conductivity in nonconductors, $\mathbf{j}$ is a current density.

\subsubsection{The model of electrodynamic processes within the MC}

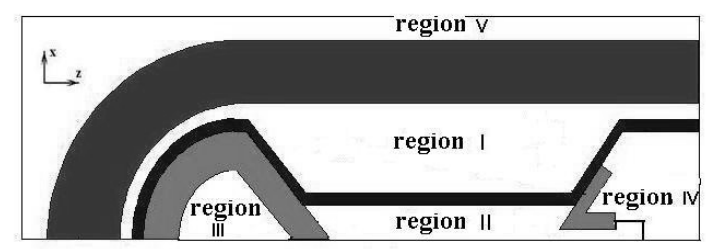

Figure 5. The calculation region for the longitudinal model.

The longitudinal section shown in Fig. 2a has the axis of symmetry therefore the calculation region is a half of the whole section (see, Fig. 5). All variables are assumed to depend only on the coordinates $x$ and $z$. The vectors of velocity and 
electric field intensity have only $x$ and $z$ nonzero components $\left(\mathbf{E}=\left(E_{x}, 0, E_{z}\right)\right.$, $\mathbf{v}=\left(v_{x}, 0, v_{z}\right)$ that lie in the plane of Fig. 5). The vector of magnetic field intensity has one nonzero $y$-component $\left(\mathbf{H}=(0, H, 0)^{T}\right)$ and it is directed perpendicular to the plane of Fig. 5 .

In the used approach the magnetic field intensity in dielectric subregions is expressed in terms of currents in the external electrical circuits. The superimposition of the magnetic field generated by the current $I_{B}$ flowing through inductor and of the magnetic field generated by the current $\left(I_{A}-I_{B}\right)$ flowing through liner leads to the following results:

$$
H= \begin{cases}-\frac{4 \pi}{l_{y}} I_{B}, & \mathbf{r} \in I, \\ -\frac{4 \pi}{l_{y}} I_{A}, & \mathbf{r} \in I I, \\ 0, & \mathbf{r} \in I I I, I V, V .\end{cases}
$$

Subregions I, II, III, IV, V are shown in Fig. 5.

The following equation for magnetic field intensity in conductors is obtained from the system (3.1) (more information in [7]):

$$
\nabla \cdot\left(\frac{1}{4 \pi \sigma} \nabla H\right)-H \nabla \cdot \mathbf{v}=\frac{d H}{d t} .
$$

The boundary conditions are either the continuity conditions $H$ at the transition from a dielectric to a conductor (from one conductor to another conductor) or the conditions of zero normal derivative. Zero initial condition is assumed.

\subsubsection{The equations of external electrical circuits}

The equations of external electrical circuits for the longitudinal model are

$$
\begin{aligned}
& L_{A} \frac{d I_{A}}{d t}+\frac{8 \pi}{l_{y}} \frac{\left(S_{I I} I_{A}\right)}{d t}+R_{A} I_{A}-U_{A}+2 \int_{\Gamma_{I I}} \mathbf{E} d \mathbf{l}=0, \\
& C_{A} \frac{d U_{A}}{d t}=-I_{A}, \\
& L_{B} \frac{d I_{B}}{d t}+\frac{8 \pi}{l_{y}} \frac{\left(S_{I} I_{B}\right)}{d t}+R_{B} I_{B}-U_{B}+2 \int_{\Gamma_{I}} \mathbf{E} d \mathbf{l}=0, \\
& C_{B} \frac{d U_{B}}{d t}=-I_{B} .
\end{aligned}
$$

Here $L, R, C$ are inductance, resistance and capacitance in the circuit, $I$ and $U$ are the current and the voltage at the capacitor coatings, $S_{I}$ and $S_{I I}$ are the areas of corresponding subregions in Fig. $5, \Gamma_{I}$ and $\Gamma_{I I}$ are their boundaries, coefficient 2 accounts for the symmetry of the region. The problem is completed by the statement of initial conditions for currents and voltages.

\subsection{The mathematical models of liner movement}

The insufficiency of experimental data leads to the uncertainty of the choice of the liner movement model. Therefore three models describing the liner material 
are used in this paper: in the first model liner is considered as a thermoelastic solid, in the second model liner is considered as a viscous incompressible liquid and in the third one liner is considered as an elasto-plastic solid.

The coupled electro-thermal-mechanical problems are very complicated. A set of different deformable body models for corresponding problems are discussed in [15] including the thermoelastic body models, the elastico-viscous body models and the elasto-plastic body models. In these models body deformations are assumed to be small (within several percents). In our problem the liner deformations are large (dozens of percents). Such problems are called geometrically nonlinear. The various thermoelastic body models considering finite deformations are presented in $[4,11,16]$. A number of large strain plasticity models are discussed in $[2,3,11,13,16]$.

\subsubsection{The mathematical model of thermoelastic liner}

In this model the liner material is regarded as an isotropic compressible thermoelastic solid $[11,12,15]$. The following symbols are introduced: $\rho$ and $\rho_{0}$ are current and initial material densities, $x_{i}$ and $a_{i}$, are Euler coordinates $\left(x_{1}=x\right.$, $x_{2}=z$ ) and total Lagrange coordinates, $u_{i}=x_{i}-a_{i}$ are displacement vector components. The following motion equations are used:

$$
\rho_{0} \frac{\partial^{2} u_{i}}{\partial t^{2}}=\frac{\partial L_{i j}}{\partial a_{j}}+\frac{\rho_{0}}{\rho} F_{i}
$$

where $\mathbf{F}=\mathbf{j} \times \mathbf{H}$ is the Lorenze force acting on the liner, $L_{i j}$ is the Lagrange stress tensor. It is defined as the following formulation for linear-elastic material (for more information see [11]):

$$
L_{i j}=\frac{\partial x_{i}}{\partial a_{k}}\left[2 \mu \gamma_{k j}+\left(\lambda I_{1}-\beta\left(T-T_{0}\right)\right) \delta_{k j}\right]
$$

where $\lambda$ and $\mu$ are the Lame coefficients, $\beta=(3 \lambda+2 \mu) \alpha_{T}, \alpha_{T}$ is the coefficient of thermal linear expansion. The strain tensor and its invariants are defined as

$$
\gamma_{k l}=\frac{1}{2}\left(\frac{\partial u_{k}}{\partial a_{l}}+\frac{\partial u_{l}}{\partial a_{k}}+\frac{\partial u_{m}}{\partial a_{k}} \frac{\partial u_{m}}{\partial a_{l}}\right), \quad I_{1}=\gamma_{i i}, \quad I_{2}=\gamma_{i j} \gamma_{i j}
$$

Herein and after the repeated index convention for summation is systematically used.

The large deformations are formed in the liner belts therefore square terms of strain tensor are taken into account. The following heat balance equation is used $[4,15]$ :

$$
\rho_{0} c_{\gamma} \frac{\partial T}{\partial t}+\beta T \frac{\partial I_{1}}{\partial t}=\nabla \cdot(\kappa(T) \nabla T)+\frac{\rho_{0}}{\rho} \phi
$$

where $c_{\gamma}$ is the unit-mass heat capacity for constant deformation, $\kappa(T)$ is the coefficient of heat conductivity, $\phi=\mathbf{j} \cdot \mathbf{E}$ is the heat generation power. 


\subsubsection{The mathematical model of liquid liner}

The system of Navier-Stokes equations is used here for describing viscous incompressible liquid movement(in terms of Euler coordinates) [15]:

$$
\begin{aligned}
\rho\left(\frac{\partial \mathbf{v}}{\partial t}+(\mathbf{v} \cdot \nabla) \mathbf{v}\right) & =-\nabla p+\eta \nabla^{2} \mathbf{v}+\mathbf{F}, \\
\nabla \cdot \mathbf{v} & =0 .
\end{aligned}
$$

Here $p$ is the hydrodynamic pressure, $\eta=\rho \nu$ is the coefficient of dynamic viscosity, $\nu$ is the coefficient of kinematic viscosity.

The heat balance equation for this model is

$$
\begin{gathered}
\rho c_{\gamma}\left(\frac{\partial T}{\partial t}+(\mathbf{v} \cdot \nabla) T\right)=\nabla \cdot(\kappa(T) \nabla T)+\phi \\
\phi=\mathbf{j} \cdot \mathbf{E}+\eta\left(2\left(\frac{\partial v_{x}}{\partial x}\right)^{2}+\left(\frac{\partial v_{x}}{\partial y}+\frac{\partial v_{y}}{\partial x}\right)^{2}+2\left(\frac{\partial v_{y}}{\partial y}\right)^{2}\right) .
\end{gathered}
$$

Here $\phi$ is the energy generation power including the Joule heat and the heat source due to viscosity.

\subsubsection{The mathematical model of elasto-plastic liner}

The liner belt is under load that exceeds the aluminum yield stress; therefore, the models of thermoelastic liner and liquid liner are approximated and the model of elasto-plastic liner is considered too. The deformation curve obtained as a result of experiments is used in the model. The analysis of liner flow near the prismatic support demonstrates [5] that the belt stretching is centered near the contact region. Therefore the large plastic deformations are formed in the liner belts. The theory of large elasto-plastic deformations presented in the paper [11] is used here. The motion equations for this model are [11, 12]:

$$
\rho \frac{\partial^{2} u_{i}}{\partial t^{2}}=\frac{\partial}{\partial \tilde{a}_{k}}\left(S_{i k}+S_{k j} \frac{\partial u_{i}}{\partial \tilde{a}_{j}}\right)+F_{i} .
$$

In contrast to equations (3.3), equations (3.5) are written in terms of updated Lagrange coordinates ( $\tilde{a}_{i}$ are UL-coordinates), $S_{i j}$ is the second PiolaKirchhoff stress tensor. In each instant of time UL-coordinates $\tilde{a}_{i}$ coincide with Euler coordinates $x_{i}$. The distinctions between these coordinates become apparent in the usage of different rates of variables change. For the UL approach the substantial derivatives are considered, for the Euler approach the local derivatives are considered.

The chosen model of large elasto-plastic deformations is based on the following assumptions $[11,12]$ :

1) The strain velocity tensor $\mathbf{V}$ (with the components $V_{i j}=\frac{1}{2}\left(\frac{\partial v_{i}}{\partial x_{j}}+\frac{\partial v_{j}}{\partial x_{i}}\right)$ ) is the sum $\mathbf{V}=\mathbf{V}^{\mathbf{e}}+\mathbf{V}^{\mathbf{p}}$ of elastic part $\mathbf{V}^{\mathbf{e}}$ and plastic part $\mathbf{V}^{\mathbf{p}}$.

2) The defining relation of elasto-plastic solid is

$$
\mathbf{s}^{H}=C^{E}: \mathbf{V}^{\mathbf{e}}=C^{E}:\left(\mathbf{V}-\mathbf{V}^{\mathbf{p}}\right),
$$


where $\mathbf{s}^{H}$ is the Hill derivate of the Cauchy stress, $C^{E}$ is the tensor of stiffness coefficients for isotropic body, the symbol ":" means double sum for repeated index (in the index form for this equation).

3) The plastic part of strain velocity tensor is defined from the following plastic flow law:

$$
\mathbf{V}^{\mathbf{p}}=\tilde{\lambda} \frac{\partial f_{y}}{\partial \mathbf{s}}=\tilde{\lambda} \mathbf{s}^{\prime}
$$

where $\tilde{\lambda}$ is an unknown parameter (the method of additional strains is used in calculations, therefore it is not necessary to find the explicit form of $\tilde{\lambda}), f_{y}$ is the yield function, $\frac{\partial f_{y}}{\partial \mathbf{s}}$ is the vector in the space of components of stress tensor deviator which is directed normally by the yield surface.

The liner material is considered to have isotropic hardening therefore the following yield function is used $[11,15]$ :

$$
f_{y}\left(\mathbf{s}^{\prime}, \bar{\epsilon}^{p}\right)=3 J_{2}\left(\mathbf{s}^{\prime}\right)-s_{y}^{2}\left(\bar{\epsilon}^{p}\right)
$$

where $J_{2}\left(\mathbf{s}^{\prime}\right)=\frac{1}{2} s_{i j}^{\prime} s_{i j}^{\prime}$ is the second invariant of stress tensor deviator $\left(s_{i j}^{\prime}=\right.$ $\left.s_{i j}-\frac{1}{3} s_{k k} \delta_{i j}\right), \bar{\epsilon}^{p}$ is the hardening parameter characterizing the magnitude of accumulated plastic deformations, $s_{y}$ is the material yield stress.

At this stage the elasto-plastic model doesn't involve the effects connected with the temperature variations.

\subsubsection{The boundary conditions statement for liner}

The conditions of free boundary are chosen (external surface forces are equal to zero). On the boundaries corresponding to the axis of symmetry the velocity component which is perpendicular to this boundary is equal to zero (the boundary condition for another component is the condition of free boundary).

The following variants of boundary conditions are used for modelling the contact between liner belt and the support:

1) adhesion conditions: $v_{x}=v_{z}=0$;

2) Coulomb friction law [11]: $\left|t_{\tau}\right|=\mu_{d}\left|t_{n}\right|$, where $t_{\tau}$ defines distributive contact tangential force, $t_{n}$ defines distributive contact normal force, $\mu_{d}$ is the dynamic friction coefficient.

\section{The Numerical Model}

The finite element method with the linear elements is used for the problem of discretization. Preliminary the region triangulation is carried out. For the longitudinal model the grids are introduced only in conductors (liner, inductor, supports) because in dielectric subregions magnetic field intensity is expressed in terms of currents in the external electrical circuits. For the transverse model the grid is introduced in the whole region, it is refined near the liner [9].

The numerical models of electro-magnetic fields and of thermoelastic liner movement are presented in the papers $[6,7]$, the numerical model of elastoplastic liner movement is analyzed in the paper [12].

In the numerical model of liquid liner movement the discrete analogs of the Navier-Stokes equations (3.4) are considered as one matrix equation with 
respect to the three-component variables vector (two components of velocity vector and pressure). The second-order finite elements are used to calculate the correct pressure value. The penalty function method is applied to realize the kinematic conditions at contact points [11].

At each time step the internal iteration cycles for electrodynamic problem, for kinematic problem and for heat problem are executed one after another. For the entire system the external iteration cycle is carried out.

\section{The Physical Parameters}

Here we present some important parameters in dimensionless form. The dimensions of the device in accordance with Fig. $2 \mathrm{~b}$ and Fig. 5 are the following: the height of inductor is 1.5 , its half-width is 1.25 . The height of liner is 0.02 , its half-width is 1.05 , its length is 6 . The distance between liner and inductor at the initial time moment is 0.02 . Initial height of liner lower edge is 0.96 . The support canting angle is $\pi / 4$.

The parameters of the inductor circuit are as follows: $L_{B}=20.0, C_{B}=$ $3.1 \cdot 10^{-5}, R_{B}=120$. The initial voltage on the capacitor is 2000 , the initial current is 0 . The parameters of the liner circuit are as follows: $L_{A}=2.5$, $C_{A}=1000, R_{A}=10^{-5}$. The initial voltage on the capacitor is 0 , the initial current is 0 . The parameters of the liner material are as follows: the Lame coefficients for the thermoelastic liner are $\lambda=1097, \mu=672$, the viscosity for liquid liner is $\nu=0.1$, the yield stress for the elasto-plastic liner is $s_{y}=0.9$, maximal stress $s_{\max }=1.875$.

\section{The Calculations Results}

The software package was developed to solve the problem. Hereafter the calculation results (in dimensionless form) of different problem variants are presented. The graphic data about the calculated solutions are demonstrated. They permit to conclude about qualitative and quantitative characteristics of the solution. The calculation results for elasto-plastic liner model with modified deformation curves are discussed in papers [8, 9, 12].

\subsection{The longitudinal model: calculation without liner circuit closing}

At the beginning, the calculations without liner circuit closing are carried out in order to examine the liner movement for different models. In these calculations the prismatic supports material is assumed to be a nonconductor. Under the given conditions the magnetic field between liner belts is equal to zero (due to the specific character of the longitudinal model described in (3.2)). The calculation region is a fourth part of the whole section, the system effective length along $y$ axis is $l_{y}=3.0$. 


\subsubsection{The thermoelastic liner}

A number of calculations with different support canting angles and contact conditions is conducted.
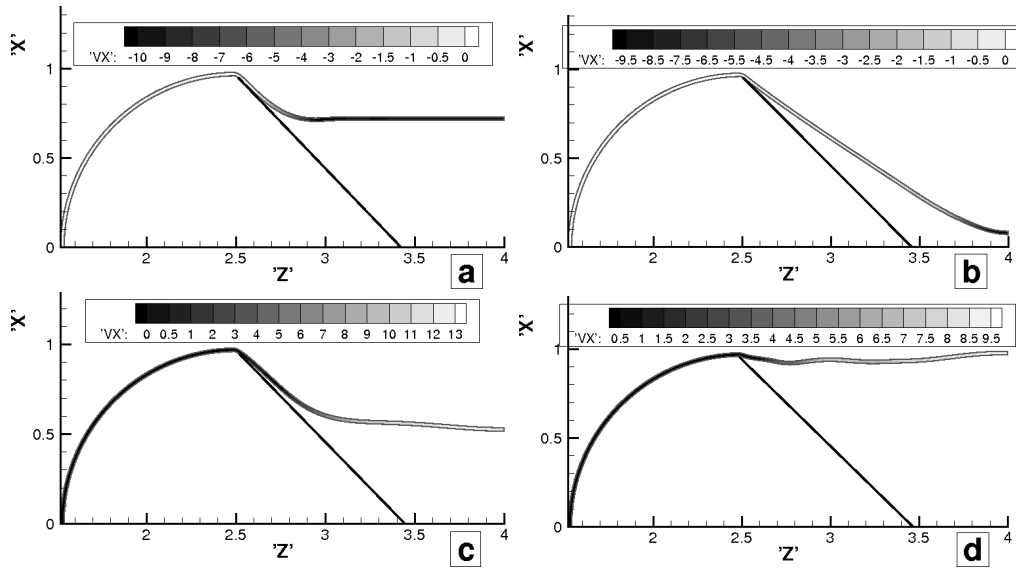

Figure 6. The thermoelastic liner positions for different times $(t=0.061 ; 0.117 ; 0.172$; $0.215 \mathrm{~ms})$.

Calculation 1. In this calculation the support canting angle is equal to $\pi / 4$, the adhesion contact conditions are chosen. The elastic liner movement is demonstrated in Fig. 6 (gray shade corresponds to $v_{x}$ ). In this calculation the liner belt does not arrive to the region bottom boundary (does not contact with another belt moving opposite to it) and it starts moving backwards (two lower figures). The elastic disturbance waves are extended across the liner. The belt adheres to the top part of the support but the new contact points do not arise during the progress of liner movement. The liner braking and its subsequent upward motion are caused by the elasticity forces. The comparison between Fig. 6 and Fig. 3a leads to the conclusion that the results of thermoelastic liner model are not in accord with the experimental data.

Calculation 2. In this calculation the support canting angle is equal to $\pi / 4$, but the contact condition is the sliding motion with the friction. The liner positions for different times are presented in Fig. 7. The liner belt contacts with the support and starts to slide along it. The belt is stretching in motion but this stretching is rather proportional relatively to the liner segment situated on the support. The calculations are stopped when the belt arrives to the bottom boundary of the region, i.e. it contacts with another belt moving opposite to it.

Calculation 3. In this calculation the support canting angle is equal to $\pi / 6$, the contact condition is the sliding motion with the friction. The liner positions for different times are presented in Fig. 8. 

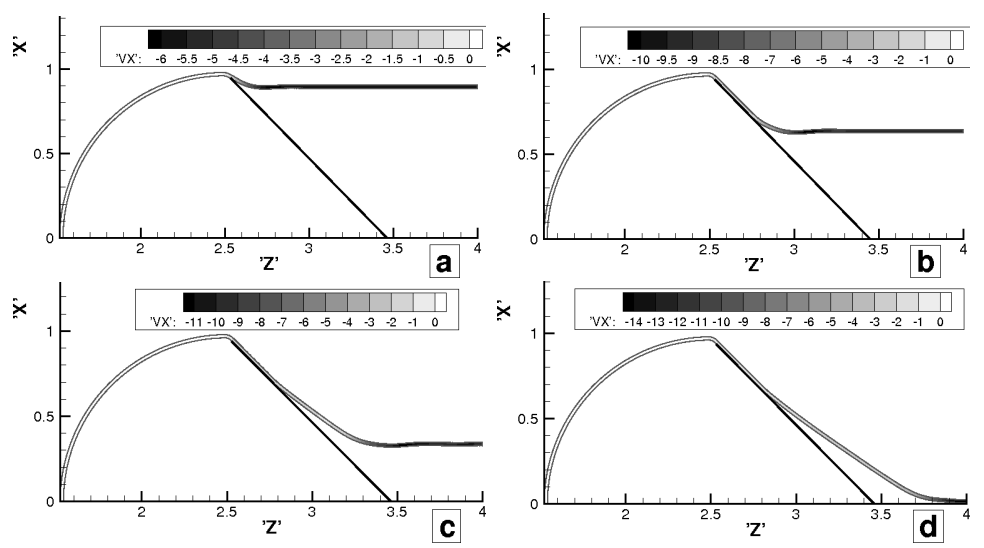

Figure 7. The thermoelastic liner positions for different times $(t=0.048 ; 0.072 ; 0.105$; $0.136 \mathrm{~ms})$.
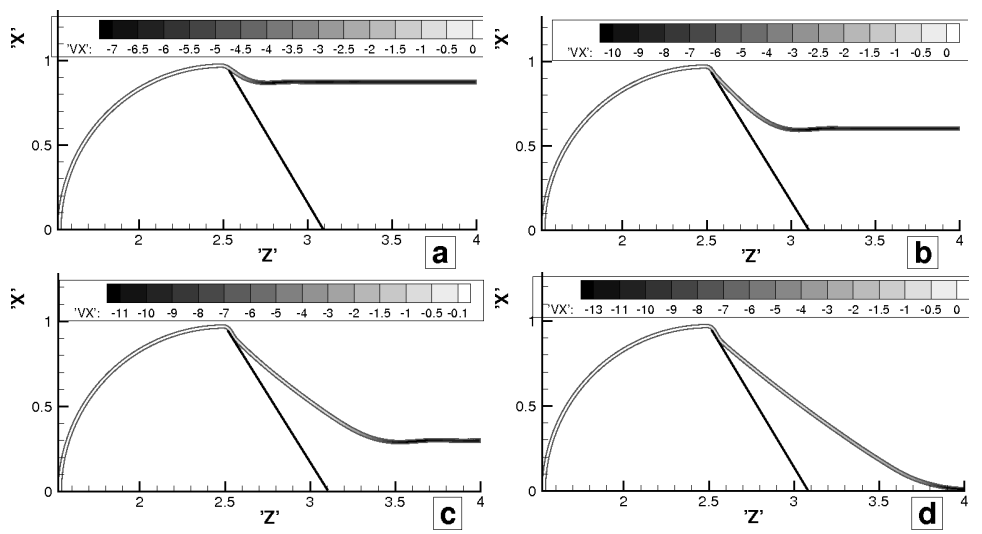

Figure 8. The thermoelastic liner positions for different times $(t=0.048 ; 0.072 ; 0.105$; $0.141 \mathrm{~ms})$.

The comparison of Fig. 7 and Fig. 8 leads to the conclusion that the decrease of the support canting angle results in the decrease of the liner segment that contacts the support. If the adhesion contact conditions are chosen, i.e., the support canting angle is equal to $\pi / 6$, the received results are identical to the results of calculation 1 .

\subsubsection{The liquid and elasto-plastic liner}

Let's discuss the results of the calculations for liquid and elasto-plastic liner with the adhesion conditions. A belt segment adheres to support surface and centre part of liner moves in a plane-parallel motion (liquid liner moves faster than elasto-plastic liner). It corresponds to the experimental data (Fig. 3). 

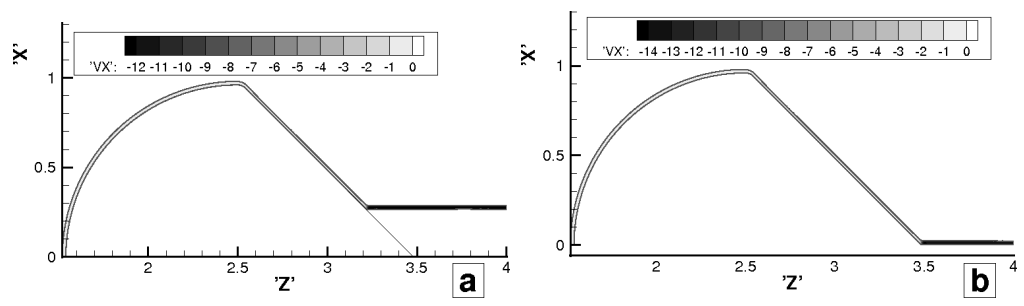

Figure 9. The elasto-plastic liner positions for different times $(t=0.092 ; 0.124 \mathrm{~ms})$.
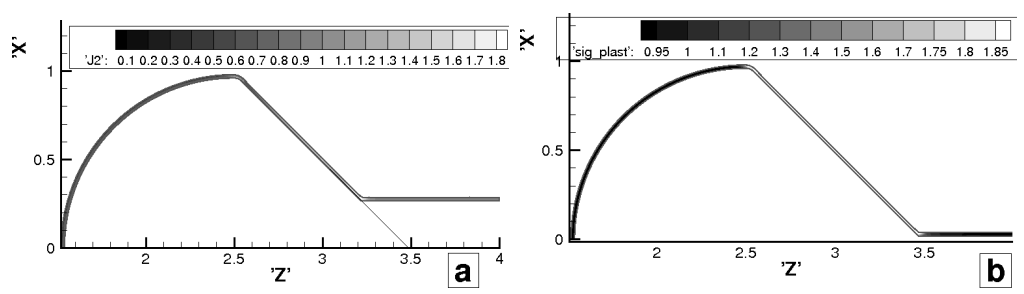

Figure 10. (a) the distribution of the stress tensor intensity $(t=0.092 \mathrm{~ms})$, (b) the distribution of the yield stress $(t=0.124 \mathrm{~ms})$.

The liner positions for two times are presented in Fig. 9.

The data characterizing the liner stress and strain state at the end of movement are presented in Fig. 10. They are the distribution of the stress tensor intensity $s_{u}=\sqrt{\frac{3}{2} s_{i j}^{\prime} s_{i j}^{\prime}}$ and the distribution of the yield stress $s_{y}\left(\bar{\epsilon}^{p}\right)$ depending on the accumulated plastic deformations [11, 15].

The distribution of the stress tensor intensity (Fig. 10a) demonstrates that the maximal stresses in the liner belt are centered near the moving boundary of the contact (as was predicted in the paper [5]). The distribution of $s_{y}$ (Fig. 10b) corresponds the maximal value of $s_{u}$ for all calculation time. The value of $s_{y}$ on the belt segment strained along support is equal to the maximum possible one for this material stress value $\mathbf{s}_{\max }$. The maximal plastic deformations are formed on this belt segment. The stress does not exceed the initial yield stress at the other belt parts and any plastic deformations are not formed.

If the contact condition of the sliding motion is chosen (more information in [8]), there are too large deformations on the belt segment strained along the support; therefore, the liner is broken.

\subsection{The longitudinal model: calculations with liner circuit closing}

For these calculations the modelling region corresponds to Fig. 5, the left support is a conductor, the right support has the conductive plate (its height is a half of the support height). The liner circuit is closed when the belt reaches the conductive plate. In this variant the calculations are carried out for the elasto-plastic liner on the basis of the analysis of the results discussed in the 
above sections.

For the selected domain the liner circuit is closed at time $t=0.0866$. If at this moment the voltage on the capacitor $\left(U_{A 0}\right)$ is equal to zero then the liner circuit current reaches the lower values (in comparison with the inductor circuit current). It is necessary to mention that the generated magnetic field is able to round the liner belt and to penetrate from the subregion I to the subregion II (Fig. 5) both in the experimental launchings and in the calculations for the transverse section. Therefore the magnetic flow pinning from the acceleration circuit is used for the liner braking [5]. The similar pinning does not take place in the longitudinal model. For the magnetic field to penetrate to the subregion II it necessary for this field to diffuse through the liner belt. This fact explains quite low values of the liner circuit current.
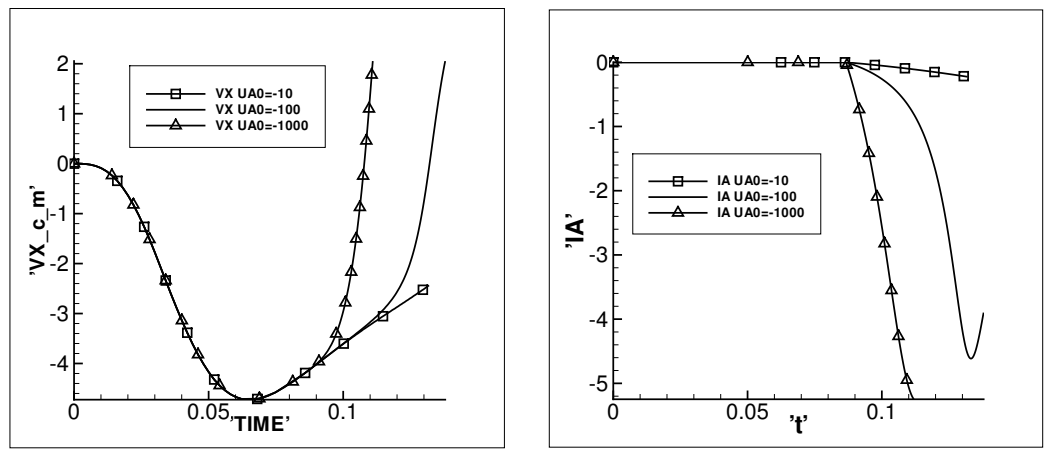

Figure 11. (a) the liner centre of mass velocity; (b) the liner circuit current.

Nevertheless it is possible to describe the belt braking by the magnetic field within the longitudinal model. For this purpose, it is necessary to generate the high-power magnetic field in the subregion II. This field will be compressed by the liner belts. To demonstrate this fact the calculations are conducted with the following liner circuit parameters: $L_{A}=0.25$, the initial voltage for different variants is equal to $U_{A 0}=-10,-100,-1000$. The diagrams of the concentrated parameters are demonstrated in Fig. 11. They are the temporal variations of the liner centre of mass velocity $\left(v_{x}\right)$ and the liner circuit current $\left(I_{A}\right)$.
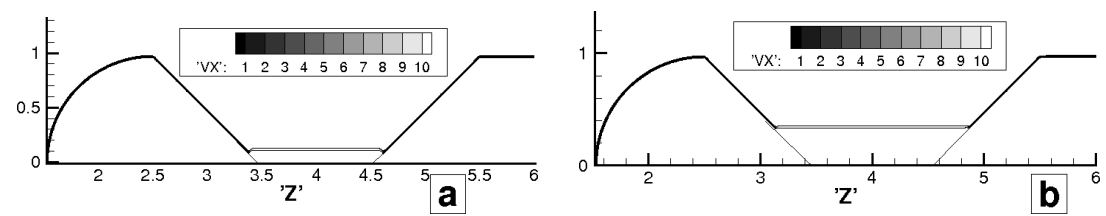

Figure 12. The liner positions in the time of calculation stop: (a) $U_{A 0}=-100(t=0.139$ $\mathrm{ms})$; (b) $U_{A 0}=-1000(t=0.113 \mathrm{~ms})$.

In the second and the third calculations the liner circuit current exceed 
the inductor circuit current; therefore the belt is completely stopped by the pressure of the compressed magnetic field (after that these calculations are stopped). In Fig. 12 the liner positions at the time of calculation stop are shown: Fig. 12a corresponds to the calculation with $U_{A 0}=-100$, Fig. 12b corresponds to the calculation with $U_{A 0}=-1000$. For the second calculation the liner belt is braked well before. In such a manner it is possible to manage the liner acceleration and braking process in the longitudinal model by controlling the parameters values.

\subsection{The transverse model}

The following results are obtained for the calculations without liner circuit closing (more information in papers [9, 12]):

1) The integral characteristics (the liner centre of mass velocity and Xcoordinate, the acceleration force, the circuit currents) correspond to one another for the different models.

2) The calculated liner form highly depends on the selected model: the elastic waves are extended along the elastic liner, the centre part of the elastoplastic liner and liquid liner moves in a plane-parallel motion, the liner width decreases at the expense of the deformations of the liner edges (the edges are deformed differently for each model). The pinch effect explains such large deformations.

\section{Conclusion}

The process of the liner electrodynamic acceleration and braking in the magnetic compressor is presented in this paper. The spatial two-dimensional approach corresponding the longitudinal section is considered (the approach corresponding the transverse section is analyzed in the papers $[5,9])$. The mathematical and numerical models describing the liner material as a thermoelastic solid, an elasto-plastic solid and a viscous incompressible liquid are briefly discussed. These models are presented in more detail in the papers $[6,7,12]$.

The main objective of this paper is the comparison of the different models and the correlation of the calculation results with the experimental data. The executed analysis leads to the following conclusions:

1) The movement of the liquid liner and the elasto-plastic liner correlates with the experimental data shown in Fig. 3. In the longitudinal section the liner belt contacts with the prismatic supports, the centre part of the belt moves in a plane-parallel motion and has small longitudinal bending. The results of these models have the qualitative similarity but they also have some quantitative differences. For example the liquid liner velocity is a little greater than the elasto-plastic liner velocity.

2) The elastic liner movement does not correlate with the experimental data. The centre part of the belt does not move in a plane-parallel motion in the calculations with the different support canting angles and with the different contact conditions. The elastic waves are extended along the liner. 
3) The magnetic field pinning from the acceleration circuit does not take place for the longitudinal model by reason of the geometrical features of the calculation region. In order to simulate the electromagnetic braking of liner one can artificially produce the magnetic field at the moment of the liner circuit closing.

\section{References}

[1] E. Azizov, S. Alikhanov, Ye. Velikhov, M. Galanin, V. Glukhih and Ye. Grabovskii. Project "Baykal" - The development of a scheme of generation of electrical pulse. VANT (Questions of atomic science and technology). Ser. Thermonuclear syntheses, 1(3):3-17, 2001.

[2] K. Bathe and M. Kojic. Inelastic Analysis of Solids and Structures. SpringerVerlag, New York, 2005.

[3] K. Bathe and F. Montans. Towards a model for large strain anisotropic elastoplasticity. Computational Plasticity, 7:13-36, 2007. http://dx.doi.org/10.1007/978-1-4020-6577-4_2.

[4] D. Blend. The Nonlinear Dynamic Theory for Elasticity. Mir, Moscow, 1972.

[5] M. Galanin and A. Lototsky. Modelling the liner acceleration and deceleration in devices of power peaking. Radiotekhnika and Electronika, 50(2):256-264, 2005.

[6] M. Galanin, A. Lototsky and V. Levashov. The Calculation of Electromagnetic Acceleration of Flat Plates in the Laboratory Magneto - Cumulative Generator. KIAM, Moscow, 2001. (prepr. of KIAM of RAS)

[7] M. Galanin, A. Lototsky and A. Rodin. The Mathematical Simulation of Liner Electromagnetic Acceleration in Different 2 Dimensional Approaches. KIAM, Moscow, 2007. (prepr. of KIAM of RAS)

[8] M. Galanin, A. Lototsky and A. Rodin. The Mathematical Simulation of Liner Movement in the Longitudinal Section of Magnetic Compressor. KIAM, Moscow, 2009. (prepr. of KIAM of RAS)

[9] M. Galanin, A. Lototsky, A. Rodin and I. Scheglov. The liner movement in the transverse section of magnetic compressor. Vestnic MGTU, 1(2):65-84, 2010.

[10] M. Galanin and Y. Popov. Quasistationary Electromagnetic Fields in Nonhomogeneous Media. Nauka, Moscow, 1998. (in Russian)

[11] S. Korobeinikov. The Nonlinear Deformation for Solids. SO RAN, Novosibirsk, 2000. (in Russian)

[12] A. Rodin. The Model of Plastic Liner Movement in Magnetic Compressor. KIAM, Moscow, 2009. (prepr. of KIAM of RAS)

[13] J. Simo and T. Hughes. Computational Inelasticity. Springer-Verlag, New York, 1998.

[14] I. Tamm. The Foundation of Electricity Theory. Nauka, Moscow, 1989. (in Russian)

[15] V. Zarubin and G. Kuvirkin. The Mathematical Models of Continuum Mecanic and Electrodynamics. Moscow State Technical University (MGTU), 2008. (in Russian)

[16] O. Zienkiewicz and R. Taylor. The Finite Element Method, volume 2. Butterworth Heinemann, London, 2000. (fifth edition) 